\title{
A New Electronic Path to the Hospital - A Prenatal Observation System
}

\author{
Arnim Nethe and Irene Krebs \\ Brandenburg University of Technology, \\ Konrad-Wachsmann-Allee 1, \\ 03046 Cottbus, Germany \\ Arnim.Nethe@tu-cottbus.de
}

\begin{abstract}
Premature babies represent a particularly endangered group of children. At the same time, their parents are frequently traumatized by the early birth and full of fear for their child. The question which arises here now is how parents can build up a relationship to their child. This article describes a visitor system which establishes the connection between child and parents and, on the other hand, could substantially support the hospital personnel with their daily work. A clinical example for this e-health application is given. This is a faster step in the direction of e-society.
\end{abstract}

Keywords: telemonitoring, IT-system, secure access, virtual visitor system, health care, early born children.

\section{Introduction}

Telemedical applications, such as intelligent assistance systems and telemonitoring systems, and their technical implementation gain more and more importance. According to BBC Research, the global market for telemedical systems alone will annually grow to approx. 11 billion Euros till 2012 [6]. Based on current research an application for a new target group in telemedicine is presented in this paper. For this practice-oriented example only few field tests have been conducted worldwide [4, 7].

\section{Premature Infants and Ill Newborn Children}

Premature infants represent a particularly endangered group of children. At the same time their parents are frequently traumatized by the early birth and full of fear for their child. In this situation we must not leave them alone, but must help them to be able to be there for their children because especially in the first weeks of life they particularly need the attention of their parents.

About 60,000 premature babies are born in Germany per year - with rising tendency: Despite a sinking birth rate premature babies constitute an ever larger share of all newborn children. Meanwhile $8.5 \%$ of all newborn children are premature babies. Thus they form the largest children's group of patients in the Federal 
Republic. Of the 60,000 premature children per year about 8,000 children are born with a birth weight of less than $1,500 \mathrm{~g}$. Despite the early birth a large part of these children is in a good course of development. About $30 \%$ are risk patients with different forms of restriction and handicap.

In addition to this, investigations verify that the social environment is of particular importance for the optimal support of premature and ill newborn children. After early birth, parents experience a phase of severe stress, accompanied by fears, feelings of guilt and helplessness. Frequently the situation asks too much of them. It is difficult for them to establish a relationship to their child in the hospital. Incubators, medical equipment, cannulae and tubes are additional barriers.

Particularly very premature children spend a long time in the incubator in a nonnatural environment in the neonatological intensive care unit. Studies indicate that the attachment behavior of premature infants frequently exhibits uncertainties or is even disorganized, as it is often observed with abused children. Therefore a failed establishment of relationships can result in permanent impairments of the cognitive, social and emotional development of the child. The fear for the survival of the child, the concern about the health development and possible impairments as well as the own feelings of guilt of having "caused" the situation impair the basis for a stable parenthood [1].

\section{A Concept for the Improvement of the Situation}

The question which arises here now is how a mutual bond can be established and developed. In addition, the psycho-social support of the families is a significant factor which must be considered.

A possible solution can be a virtual visitor system in the neonatology intensive care unit. If parents could see the baby again the evening before bedtime, that would minimize the fears and increase the bond to the child. Thus family life can also be more relaxed since the new family member can be integrated into the family. The parents can show the child to friends and relatives and thereby integrate him/her into the family environment. In addition, the virtual visitor system would supplement the control services of the hospital personnel. It is possible for the personnel to additionally observe the child by a camera from the monitoring room. In cases when the child accidentally pulls out the tubes, the nursing personnel can react faster because this can be noticed by the live monitoring.

Thus, on the one hand, the virtual visitor system would strengthen the mutual bond between child and parents and, on the other hand, would substantially support the hospital personnel in their daily work. Thus the nurses, hospital aides and physicians can do their jobs more productively and more efficiently.

The approach of installing a prenatal monitoring system - we call it "BabyLive" in hospitals cannot be taken for granted today, although the advantages are obvious and a technical implementation would be possible without any problems. In the following part it is explained how the approach described can be implemented and which factors have to be considered. 


\section{IT Concept for the Implementation of the BabyLive System}

In an IT concept all technical prerequisites for the implementation of the project in a hospital are described. A basic prerequisite for the IT application is, among other things, the development and implementation of a uniform IT security concept with the goal of guaranteeing the integrity, confidentiality and availability of data and services, the operation of an efficient, highly-available, preferably open and safe clinical center net for the transmission of digital data of all kinds as well as the build-up and operation of a need-oriented support structure.

In addition to the basic equipment of the workplaces, the users expect that a number of superordinate IT services are provided with high availability and reliability. This includes, among other things, a central listing service for purposes of user, assigning the parts, the rights management, a network server for the supply of the personal computers, secured access to the clinical center net, IT security checks, as well as reactive and proactive IT safety precautions (e.g. firewall, intrusion detection, intrusion prevention, virus defense, spam defense), email services as well as videoconference services (video transmission of the child's photographs).

\subsection{Services in the IT Environment}

In connection with the basic equipment and IT services mentioned above there are also further requirements. They can roughly be divided into four groups:

- A platform for rapid informing about current events on the homepage of the clinical center and information about the further development of BabyLive by means of newsletter.

- A consultation for informing parents about the use of the service and a catalog for frequently asked questions (FAQ).

- Training on general IT topics for nursing personnel and parents for using the service BabyLive.

- Technical support for the staff, analysis of the use of the service, assessment and/or planning of IT procurements as well as the procurement of software on the basis of valid contracts.

\subsection{Security in the IT Environment}

The planning phase covers the threat analysis, a risk analysis, the preparation of a safety strategy and a safety model. In the threat analysis possible dangers are determined and their causes such as organizational, technical or user-related causes are found out. These tasks can only be mastered if certain knowledge and experience in the field of IT security is available and the weaknesses of the existing systems and services are known (see Fig. 1).

In the risk analysis the determined threats are evaluated. The evaluation contains, on the one hand, the estimation of the probability of the occurrence of the threats, and, 


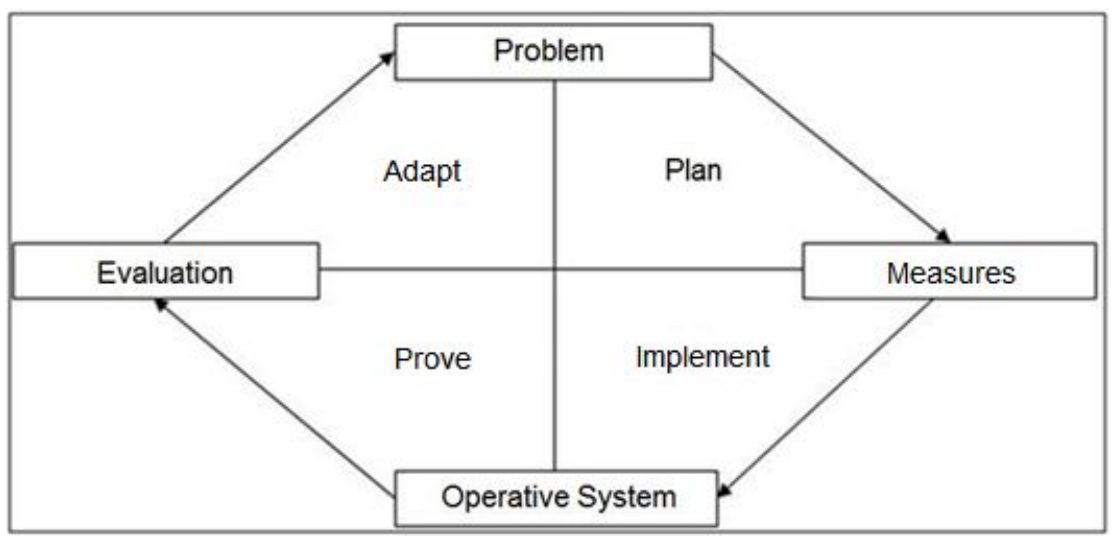

Fig. 1. Process model for the implementation of the BabyLive system [5]

on the other hand, the estimation of the caused potential damage when a threat occurs. Due to the threat and risk analysis it can be decided which safety requirements are to be set for the system to be designed. These requirements are summarized in a safety strategy. To meet the security needs general basic safety functions can be used. These functions are:

- Identification and authentification: Subjects and objects must be clearly identifiably, for example with the access to resources or to certain files. To facilitate identification characteristics for an authentification must be specified, such as passwords and a digital fingerprint.

- Rights administration: This concerns the prevention of unauthorized access to objects. Administrative rights must be specified for the objects to be protected, such as reading and writing rights.

- Rights examination: In order to implement rights administration the accesses must be controlled. This is the task of rights examination. For this the time and the kind of control must be specified. Further the measures to be taken in cases of unauthorized access must be regulated.

- Preservation of evidence: The preservation of evidence is to secure proof so that accomplished actions cannot to be denied later. For this it must be specified which events and information are to be logged and who possesses access to the logged information.

- Reprocessing: To retain information privacy shared operational funds must be settled before another process accesses them.

\section{Practical Example in the Pediatric Clinic of the Carl-Thiem-Klinikums (CTK) Cottbus}

The neonatology unit of the pediatric clinic of the Carl-Thiem-Klinikum Cottbus is one of four institutions of care level 1 in the Federal State of Brandenburg. Care level 
1 means that only these institutions are allowed to bill health insurance companies for the treatment of newborn children under a birth weight of 1250 grams. Further hospitals with neonatology units are located at a great geographical distance from Cottbus.

The neonatology unit in Cottbus is divided into two sections. In the intensive room for premature infants in the delivery room ill premature and newborn infants are primarily treated, for example those who need breathing support. The second section is the intensive care unit for premature infants, where those newborn children are cared for who do not need intensive medical treatment (any more). [2]

In all areas of the neonatology unit where premature infants are cared for, connections for hospital-internal communication are available. Furthermore, the monitors (security camera screens), which for example display the heart frequencies of the children, are all interconnected with one another. These data can be retrieved by means of administrative software at several terminals at the unit. The clinical center has its own homepage with a babysite, where only photos of newborn children have been made available so far. This site could be supplemented by an extension for retrieving video transmissions of the children. Due to the special operation of a hospital information system it is necessary to parallel implement the complete infrastructure for the BabyLive system.

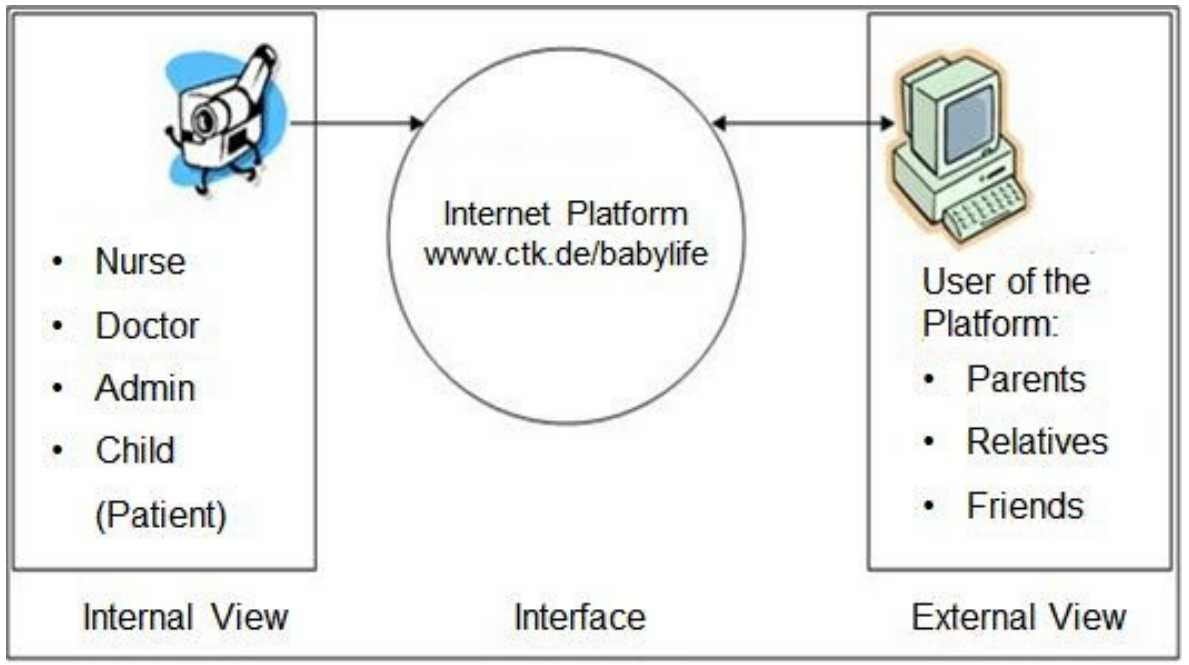

Fig. 2. Participants and use relationships

After the introduction to IT security it is now to be illustrated how internal and external security can be implemented using the pediatric clinic in the CTK as an example. For the illustration of the section which is to be considered here see figure 2 .

The internal view represents the Carl-Thiem-Klinikum Cottbus and the participants who use video transmission and can be observed by the camera. The pictures are transferred to the Internet platform. The users of the platform, such as the parents, can then access the Internet platform and the picture transmissions. The Internet platform 
is an interface between the clinical center and the platform users. Additionally to the users already specified, the nursing personnel can also use the service. The nursing personnel can track the picture transmission of all cameras on the computer in the monitoring room.

\subsection{Internal Security}

For internal security no information should get out which is not intended for the outside world. This could be, for example, the treatment of the child which is not to be filmed and thus must not become visible by the users of the platform of the virtual visitor system, but also the protection against an internal access to the system by unauthorized people.

First, possible risks for the clinical center and its personnel must be listed. These are:

- The treatment and care of the child by the nursing personnel and the physicians are filmed and transmitted.

- Staff of the clinical center could overhear the data traffic.

- A coworker can use the service of the video transmission for which s/he does not possess authorization.

- By pretending identity a coworker reroutes the data.

From the threat analysis the following protection goals result:

- Video transmission must be prevented during the treatment and nursing of the child.

- The access to the service, the servers and the data must be controlled and checked.

Before using the service the personnel must authorize themselves.

So that the nursing personnel and the physicians are not observed during the treatment of the child, the camera must either be turned off every time or the bed is put away from the camera. However, this is too difficult, costs time and especially in emergencies cannot always be implemented. Instead a sign could be attached in front of the lens of the camera, for example with the text "I am off" or "I am being fed", which is placed in front of it every time the child is not to be observed. The solution is simple, does not cost too much time and the user of the Internet platform knows what is happening in these moments.

So that the data traffic cannot be overheard by staff, it must be ensured that the net of the video transmission is operated separately from the remaining clinical center net. Thus only the staff of the neonatology unit can access and use the service of the virtual visitor system. Further, authorization before using the service can guarantee that no unauthorized persons have access to the data communication on the net and thus cannot reroute or abuse the data.

\subsection{External Security}

The ways from the Internet platform to the user of the platform and from the user to the platform are of importance for external security. Possible dangers could be: 
- Unauthorized access to the Internet platform,

- Abuse of the access to data,

- Spoofing of emails by an aggressor,

- Unauthorized reading of emails,

- Attack on communication between the interface and the PC of the user,

- Spoofing of the identity,

- Execution of malware on the web server by the user.

From the threat analysis the following protection goals result:

- Access to the service and the data must be controlled and checked.

- Before using the service the user must authenticate himself/herself.

- The integrity, authenticity and privacy of emails must be guaranteed.

- Access of the user to the clinical center's internal net must be impossible.

\subsection{Technical Realization}

For the implementation of the schematic representation of the BabyLive system shown in Figure 3 the following steps are necessary: On the Internet site of the CarlThiem-Klinikum Cottbus a link is to be established, which is to refer to the site of the BabyLive system. The link indicates the IP address of the SDSL modem of the unit. For safety reasons, an inquiry about the user name and the password will be carried out here. This authentification is global, i.e. each user of the camera platform has this registration for the front page of the cameras. The protection is organized so that no unauthorized persons can access this homepage. Furthermore, this global registration

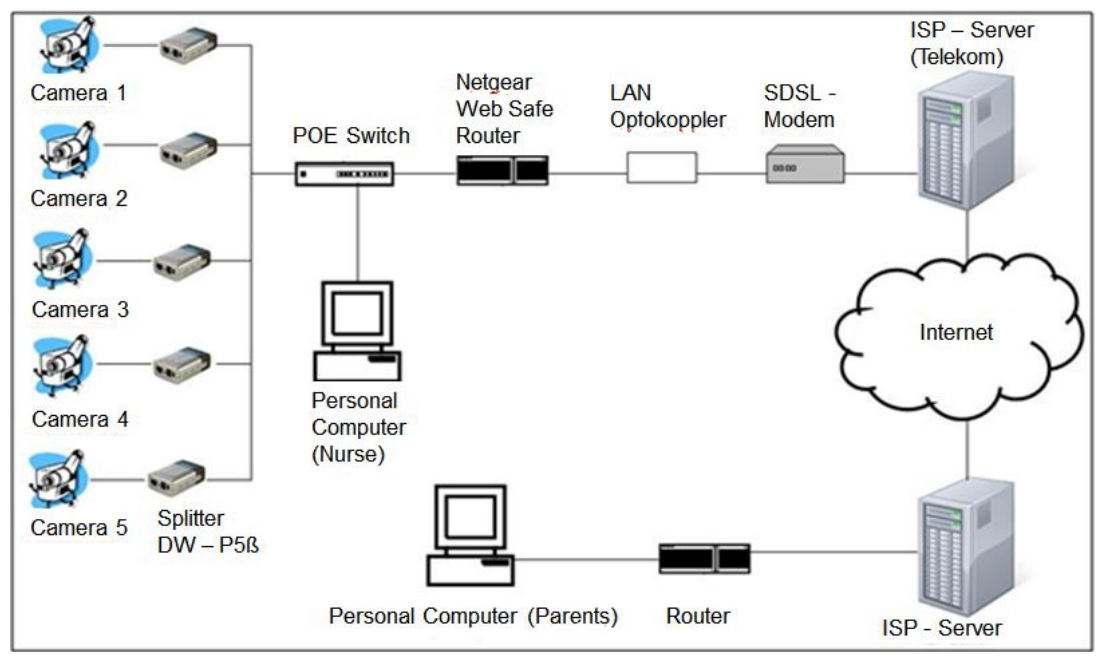

Fig. 3. Schematic representation of the BabyLive system 
is logged in a $\log$ file. This file can also be used for statistical purposes in order to determine how successfully this service is used and if further investments would be worthwhile. A camera is assigned to every child. The parents select the camera which was assigned to them. Again there will be an inquiry about the user name and the password. The router implements port forwarding to the server in the nurses' room, on which the hosting of the webpage of the camera entrances is run. The IP addresses of the cameras are mapped on the individual IP of the server by the port forwarding, so that the internal infrastructure remains transparent for the user. After the authentification the video transmission of the child becomes visible by means of a live stream point to point. This means that the video transmission is converted into a video/audio stream in real time by means of encoder software. In this way the stream can be transmitted via the Internet. Point to point streaming means that a central server provides a connection between the participants (child/parents). This data exchange is server independent. The video transmission is only stopped by logout or the end of the visiting time. The visiting time is dependent on the organization of the care of the children, as it is also usual with normal visits in the hospital. Furthermore, the visibility of the child in the net can be disturbed by certain events, for example when the child is being fed or examined. As already mentioned, in such events posters are placed in front of the camera.

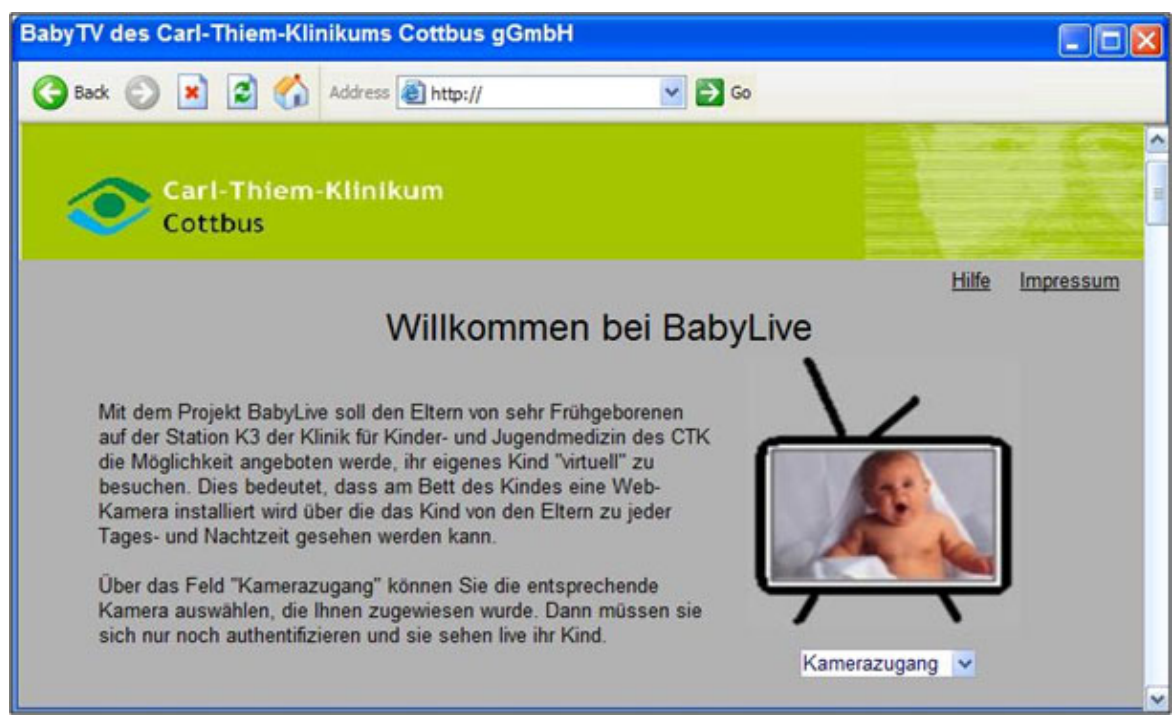

Fig. 4. Homepage of the Internet platform: "Welcome to BabyLive: With the project BabyLive parents of premature infants at the unit K3 of the hospital for child and adolescent medicine of the CTK are offered the possibility to visit their child "virtually". This means that a web camera is installed at the bed of the child via which parents can see the child at all times of the day and night. Using the field "Kamerazugang" you can select the camera which was assigned to you. Then you only have to authentificate yourselves and see your child live". 
The relatives take over a significant part of the implementation. They must make themselves familiar with the technology, receive instructions and give their agreement for the video transmission. In order to make the project a success, parents would have to accept and use this hospital service. The extent to which the service offered is used helps decide whether there should be more investment in this project.

The fear that the pictorial material could be abused by unauthorized people can be taken away by entrance identification. By this identification only authorized users of the respective camera can see the child. If the relatives forget the access code, the staff of the hospital can help by generating a new access code and a new password (see Fig. 4 and Fig. 5).

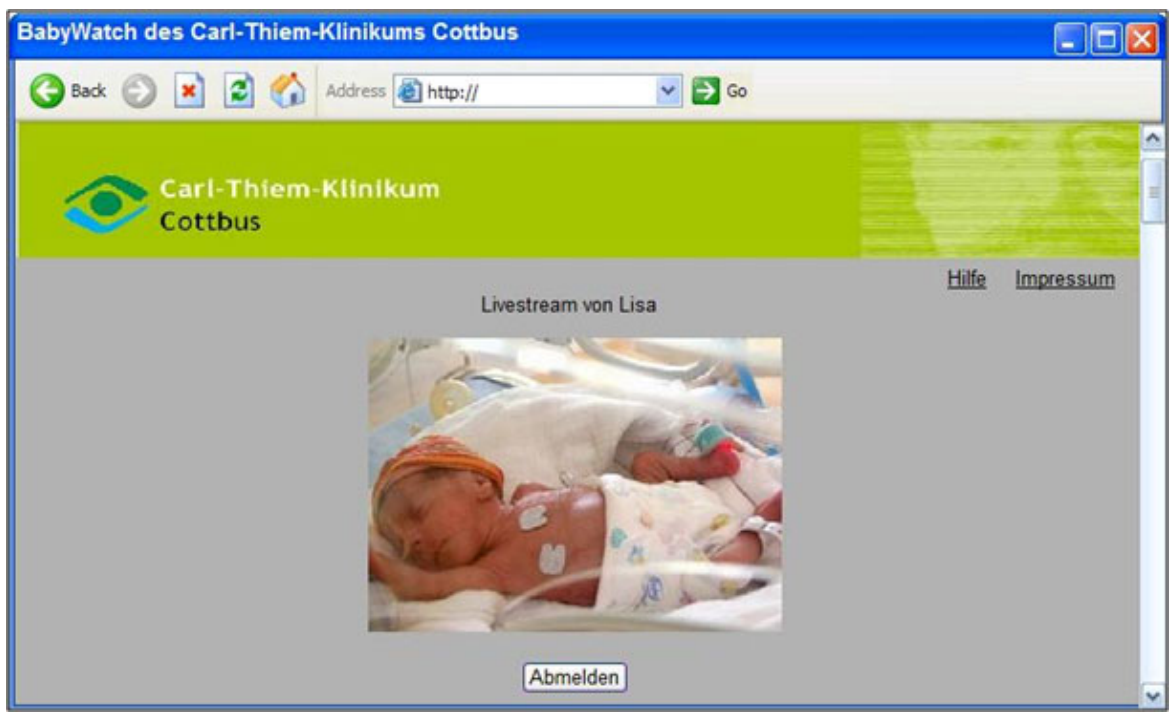

Fig. 5. Camera site of the Internet platform

\section{Conclusions}

Today there is a highly complex IT structure in every hospital. The implementation and adaption of a completely new access path with previously non-existent users for a prenatal observation system increases the complexity of this IT structure. Thus, among other things, new requirements arise regarding the development and implementation of a uniform IT security concept with the aim of guaranteeing the integrity, confidentiality and availability of data and services, organizing the operation of a highly efficient and available, preferably open and secure hospital network for the transmission of digital data of all kinds as well as organizing the setup and operation of a demand-oriented support structure. In addition, there is the training of all people involved on how to use the prenatal observation system. 
A priori a modern hospital is a part of e-health. Thus with this prenatal observation system a telemedical platform is created for the computer affine generation of young parents with which a children's hospital can significantly improve e-service and in this way can make a big step in the direction of e-society.

\section{References}

1. Kinderhilfe, D.: Das Frühförderprogramm der Deutschen Kinderhilfe (FFP), Deutsche Kinderhilfe e.V. (2007)

2. Erler, T., Ellsäßer, G.: Die Gesichter der Opfer - Auswirkungen von Gewalt bei Kindern und Jugendlichen erkennen. Pädiat. Prax 75, S.387-S.395 (2010)

3. Krebs, I., Nethe, A.: Zugang für Alle - Barrierefreies Leben in einer digitalen Welt für eine langfristige Selbstbestimmtheit. In: Demographischer Wandel - Assistenzsysteme aus der Forschung in den Markt (AAL 2011) (2011)

4. LeDuc, K., Haley-Andrews, S., Rannie, M.: An observation unit in a pediatric emergency department: One children's hospital's experience. Journal of Emergency Nursing 28(5), 407-413 (2002)

5. Poguntke, W.: Basiswissen IT-Sicherheit. Das wichtigste für den Schutz von Systemen \& Daten, 2. Auflage, Witten (2010)

6. Reinhardt, P.: Potenziale für intelligente Assistenzsysteme. DeviceMed - Fachmagazin für Hersteller medizinischer Produkte 9. Jg., 5 (September 2010)

7. Vocera Communications, Inc.: Vocera Benefits Study at The Children's Hospital at Westmead, Prepared by The NTF Group March 2007 Vocera Communications (2007) 